\title{
Symmetric Walking Control: Invariance and Global Stability *
}

\author{
Sang-Ho Hyon and Takashi Emura \\ Department of Bioengineering and Robotics \\ Tohoku University \\ Aramaki-Aza-Aoba 6-6-01, Sendai 980-8579, Japan \\ sangho@ieee.org
}

\begin{abstract}
This paper first presents a novel control strategy for periodic motion control based on a Hamiltonian system. According to the strategy, hybrid symmetric orbits (ideal walking gaits) are explored using reversal symmetry of the Hamiltonian system. Then, an invariance controller, a Symmetric Walking Controller, is derived systematically to distribute the symmetric orbits densely throughout the entire phase space. Finally, a new robust walking speed controller is formulated based on the passivity of the controlled system. Consequently, solutions starting from any point globally converge to a stable limit cycle having a desired energy level. The controller has strong passivity and robustness, thereby rendering it capable of using external disturbances as energy for walking propulsion. It requires no model parameters and can be implemented in a very small program size. Furthermore, it is applicable to any biped robot without major modification. In this report, the effectiveness of this controller is proved mathematically, validated numerically, and confirmed experimentally.
\end{abstract}

Index Terms-Dynamic locomotion, Hybrid periodic orbit, Invariance, Passivity, Controlled Hamiltonian, Symmetric orbit.

\section{INTRODUCTION}

\section{A. Background}

The mathematical substance of walking is nothing but a nonlinear periodic orbit. Nevertheless, this is not the periodic orbit of a single continuous dynamical system that has been studied for long time in celestial mechanics. Rather, it is the hybrid periodic orbit of a hybrid dynamical system comprising continuous dynamics and discrete dynamics, which has not been discussed well in dynamical system theory [1].

Because walking robots must always actively be prevented from falling, a theoretical guarantee of stability is of utmost importance for controller designers. Regarding this point, the ZMP criterion has played a major role in controller synthesis. ZMP can be considered as a special constraint that ensures the tracking of some pre-planned reference walking path in real-world coordinates (X-Y-Z). Therefore, in a typical ZMP-based walking controller, some walking patterns are partially designed in real-world coordinates; subsequently, the remaining patterns are calculated off-line or in real-time so that the ZMP criterion is met [2][3][4].

Because the location of ZMP restricts the walking gait, design of ZMP as well as other pre-specified walking pat-

*This study is partially supported by the Ministry of Education, Science, Sports and Culture, Grant-in-Aid for Young Scientists (B), 15760144. The authors thank M. Suzuki for machining the mechanical parts of the robots.

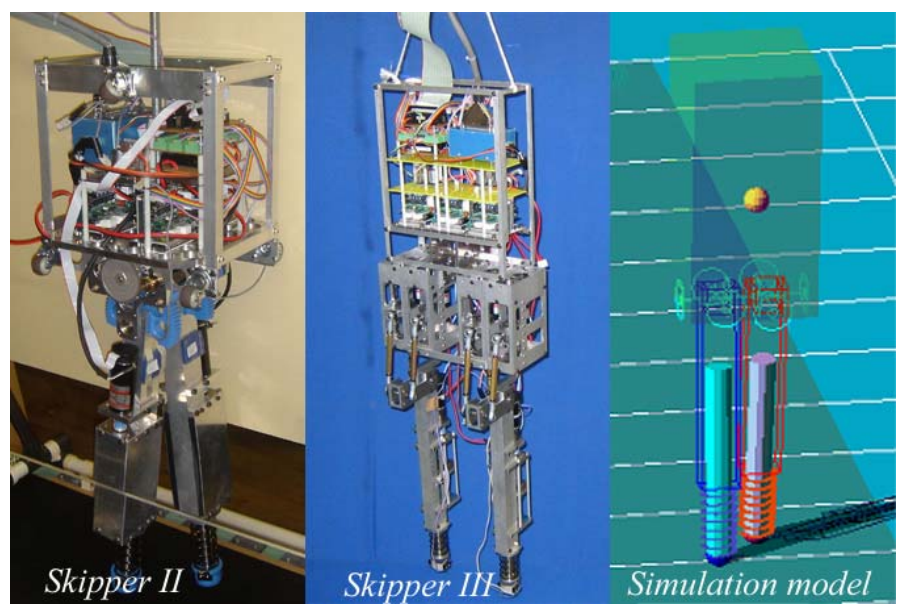

Fig. 1. Experimental and simulation models of biped walking robots

terns have remained as salient obstacles. On the other hand, momentum-based controllers that consider momenta instead of ZMP have been proposed recently [5]. Although they guarantee the regulation or tracking of a reduced inverted pendulum model, their overall gait stability depends on the walking pattern synthesis. An appropriate method to design a globally stable and natural pattern remains unresolved, as in the case of ZMP-based controller.

In that regard, passive bipedal walking robots have inspired optimal and energy-efficient walking controllers [6]. Using control inputs as the gravity effect, passive-like walking was realized on level ground [7]. However, because the controlled walking gait has the same property of the original uncontrolled passive walker on a slope, the region of attraction is not large: improved stability remains elusive.

\section{B. A novel control strategy based on Hamiltonian dynamics}

We propose a controller that generates stable and robust locomotion gaits autonomously without any pre-planned reference trajectories. To do so, we first propose a novel and general control strategy for controlling oscillatory movement based on Hamiltonian dynamics. The strategy comprises three steps:

1. First disregard dissipativity of the system to extract Hamiltonian (conservative) dynamics. Then, design an invariance controller that restricts the hybrid solution into invariant sets. 
2. Account for dissipativity and design some period and/or energy controller to obtain asymptotic stable periodic orbits (limit cycle) that pass through some desired target states.

3. Design adaptive mechanisms to minimize control efforts. The resultant controller has a hierarchical structure in which the existence, stability, and boundedness of the solution are ensured by the lowest layer with a high priority; more advanced control goals are achieved at the higher layer.

Because of the lack of space in this paper, we cannot describe details of this general control strategy. Instead we will show one specialized application of this strategy for walking control, along with an outline of the paper organization.

In Step 1, dissipativity is disregarded; thereby, the system becomes purely Hamiltonian. Symmetry of the Hamiltonian system is utilized to find a symmetric (hybrid) periodic orbits (ideal walking gaits). Section II reviews reversal symmetry of Hamiltonian dynamical systems. This property reveals the ideal walking gaits of the biped robot, as explained in Section III. Moreover, the invariance controller is introduced to enlarge the existence region of the periodic orbits. The controller renders the system passive and the hybrid solution invariant. Thus constructively derived controller based on symmetry is called symmetric walking control (SWC) [8].

In Step 2, energy control is applied to the closed-loop system obtained in Step 1 to achieve asymptotically stable periodic orbits. Specifically, Section IV-B proposes a passivity-based robust speed-controller with an additional control input. In particular, it is proved theoretically that, in principle, under $S W C$, the robot does not fall. Section V evaluates the controller by simulation or experiments using the dynamic biped robot Skipper II and Skipper III (Fig. 1). The controller is simple enough that it can be implemented on some inexpensive microcontrollers.

In Step 3, we apply an adaptive controller to minimize control inputs. It includes the problem of re-design of the actuation method, in which some passive elements such as springs may be introduced to the model. However, this is our working problem and is not applied in this paper. Section VI summarizes the effectiveness of SWC and discusses some extensions including walk-to-run control.

Note that the above general control strategy was partially applied by the authors to a 4-DOF monopedal and a 5DOF bipedal running robot in [9][10][11], in which an energy-preserving controller actively removed the dissipation at touchdown and revealed quasi-periodic orbits on torus in Step 1, a delayed-feedback controller generated orbitally stable running gaits in Step 2, and a spring stiffness adaptation minimized control inputs to obtain completely passive running (for the monoped case) in Step 3.

\section{REVERSAL SYMMETRY IN HAMILTONIAN SYSTEM}

As explained in Step 1 of the previous section, our strategy is to extract and investigate Hamiltonian dynamics. Hamiltonian mechanical systems possess a special symmetry called time-reversal symmetry [12]. Consider a diffeomorphism $F$ on manifold $\mathcal{M}$

$$
F: \mathcal{M} \rightarrow \mathcal{M}
$$

An involution $R: \mathcal{M} \rightarrow \mathcal{M} \quad(R \circ R=i d)$ is called (involutive) reversing symmetry of (1) if the following relationship holds.

$$
R \circ F=F^{-1} \circ R
$$

Let $\phi_{t}:\left(q\left(t_{0}\right), p\left(t_{0}\right)\right) \mapsto\left(q\left(t_{0}+t\right), p\left(t_{0}+t\right)\right)$ be an flow map of Hamiltonian "mechanical" system with Hamiltonian

$$
H_{0}(q, p)=\frac{1}{2} p^{T} M(q)^{-1} p+U(q) .
$$

"Any" Hamiltonian mechanical system is reversible w.r.t. involution $R:(q, p) \mapsto(q,-p)$; that is,

$$
R \circ \phi_{t}=\phi_{-t} \circ R \text {. }
$$

The intersection of the fixed point sets Fix $(R)$ and Fix $(R \circ$ $\left.\phi_{t}\right)$ is of special interest because an orbit, called a $(R$ ) symmetric orbit, passes through the intersection and the orbit is closely related to our "hybrid" periodic motion. In summary, if there is a pair of the involution $R$ and the $R$ reversible flow $\phi_{t}$, and if we can find the intersection of Fix $(R)$ and Fix $\left(R \circ \phi_{t}\right)$, then we obtain a symmetric orbit. This is an important part of our control strategy.

Time-reversal symmetry has been studied mainly in celestial mechanics [12]. The idea was firstly implicitly applied to a monoped hopping robot in [13], but recently, more concrete treatment was done in [14]. In particular, the theory can prove existence of the "controlled" quasi-periodic orbits of a 4-DOF one-legged hopper discovered in [9][14]. But the purpose here is different from these works; we derive an invariance controller and a passivity-based controller in the following two sections.

\section{SYMMETRIC WALKING CONTROL}

This section develops an invariance controller for biped robots according to Step 1 of the strategy in Section I-B.

\section{A. Symmetric (hybrid) periodic orbit of biped robot}

Consider a compass-like biped model, as shown in Fig. 2. Let $q=\left(q_{1}, q_{2}\right)$ be generalized coordinates on configuration space $\mathcal{N}:=\left\{q \in S^{2}|| q_{1} \mid \leq \pi / 2\right.$ and $\left.\left|q_{1}\right|<\left|q_{2}\right|\right\}$, where the solution can be defined. Let $z=(q, p)$ be the canonical coordinate on phase space $\mathcal{M}=T^{*} \mathcal{N}$. The conjugate momentum $p$ is associated with $q$ by $p=M(q) \dot{q}$, where

$$
\begin{aligned}
& M(q)= \\
& {\left[\begin{array}{cc}
\left(m_{1}+2 m_{2}\right) L^{2}+m_{2}(L-b)^{2} & -m_{2} b L \cos \left(q_{1}-q_{2}\right) \\
-m_{2} b L \cos \left(q_{1}-q_{2}\right) & m_{2} b^{2}
\end{array}\right] .}
\end{aligned}
$$

Define the control input as $u_{1}=\tau \in \mathcal{R}^{1}$. Then the dynamics of the biped model at the single support phase can be represented by the 4-DOF Hamiltonian control system [15]

$$
\dot{z}^{\mu}=\left\{z^{\mu}, H_{0}\right\}-\left\{z^{\mu}, H_{1}\right\} u_{1} \quad(\mu=1,2,3,4),
$$




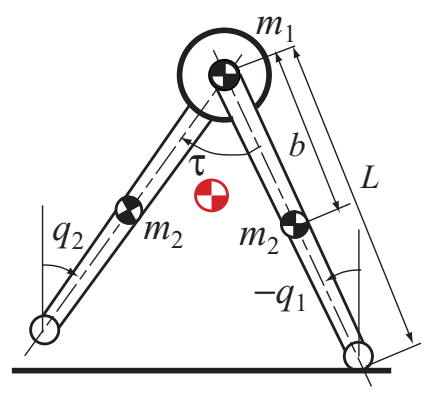

Fig. 2. A compass-like biped robot comprising a point mass: $q_{1}$ is the absolute angle of the supporting leg, whereas $q_{2}$ is that of the swinging leg. $\tau$ is the hip torque.

where

$$
\begin{aligned}
H_{0}(q, p) & =\frac{1}{2} p^{T} M(q)^{-1} p+U(q), \\
H_{1}(q) & =q_{1}-q_{2}
\end{aligned}
$$

are the internal Hamiltonian and interaction Hamiltonian, respectively, and $\{\cdot\}$ is the Poisson bracket [16]. The potential function is defined as

$$
U(q)=\left(m_{1}+2 m_{2}\right) g l \cos q_{1}-m_{2} g b\left(\cos q_{1}+\cos q_{2}\right) .
$$

Now, consider $R$-reversible "uncontrolled" flow $\phi_{t}$ of (5). Instead of conventional involution $(R:=(q, p) \mapsto(q,-p))$, here we are interested in the mirror image about $q$-axis, that is, the involution

$$
R:\left(q_{1}, q_{2}, p_{1}, p_{2}\right) \mapsto\left(-q_{1},-q_{2}, p_{1}, p_{2}\right) .
$$

Because $U(q)$ is symmetric (invariant) to $R, R$ is reversing symmetry of $\phi_{t}$.

For hybrid dynamical systems such as legged robots, $R$ reversibility of the flow is especially important when the involution $R$ itself can be considered as the discrete jump of the hybrid system. In this case, the $R$-symmetric orbit corresponds to the hybrid periodic orbit of the system (walking gait). The mirror image (9) is a natural involution for the compass-like biped robot because it meets the ideal touchdown condition $\left(q_{1}+q_{2}=0\right)$. Therefore, the connection of the involution $R$ and the $R$-reversible flow $\phi_{t}$ constitutes an "ideal" symmetric walking gait.

To render this connection make sence, one should consider the restriction of flow $\phi_{t}$

$$
F:=\left.\phi_{t}\right|_{t=T} \quad,
$$

where $T$ is defined as the time when the touchdown condition $q_{1}+q_{2}=0$ holds. With this background and the assumption of "physical" realization of $R$, the problem is reduced to finding the fixed point set $\operatorname{Fix}(F \circ R)$. Define $z^{*}=\left(q^{*}, p^{*}\right) \in$ $F i x(F \circ R)$. The system is non-integrable; therefore, we should introduce some numerical algorithm.

Figure 3(a), though coarse, depicts $F i x(F \circ R)$, which is obtained using the algorithm developed in [17]. The result indicates $F i x(F \circ R)$ forms a 2-dimensional surface. The

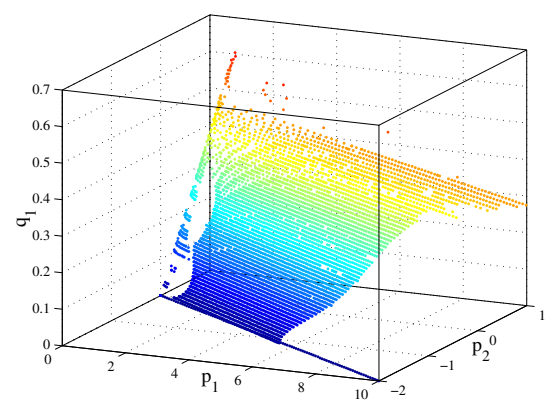

(a) Partial plots of $F i x(F \circ R)$, where $q_{2}$ is omitted because $q_{2}^{*}=-q_{1}^{*}$.
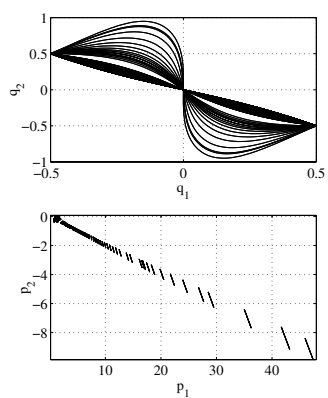

(b) Symmetric orbit for $q_{1}^{*}=0.5$
Fig. 3. Numerical results of symmetric orbits (ideal walking gait) of a compass biped with the parameters: $m_{1}=2, m_{2}=1.2, b=0.2, L=0.4$. The gaps result from roughness of the initial guess; they do not indicate emptiness.

trivial one is the origin, which corresponds to the equilibrium point. Figure 3(b) shows the phase portrait of symmetric orbits starting from a fixed point in Fig. 3(a). The symmetric orbit is mapped onto itself by $R$ with its direction reversed. A similar orbits in the top of Fig. 3(b) appear in the "ballistic walking gait" in [18]. Our examination newly obtains the following results.

(1) Symmetric orbits are at best neutrally stable (the characteristic exponents [19] lie on $S^{1}$ ).

(2) Symmetric orbits have at most three-times bifurcations. The details will be presented elsewhere. Just note that these symmetric orbits cannot be realized without control because the actual impulse equation always perturbs the initial condition away from $z^{*}$.

\section{B. Symmetric walking controller}

We have seen that uncontrolled symmetric orbits are at best neutrally stable. However, using invariance control, we can actively construct another controlled symmetric orbit reversible to $R$. Specifically, we can obtain controlled symmetric orbits by setting the output function,

$$
y=h(x)=q_{1}+q_{2},
$$

to zero and using a leg exchange scheme,

$$
S(x):=\left\{x|| q_{1} \mid-\bar{q}\right\}=0,
$$

where $\bar{q} \geq 0$ is the switching angle (scalar value), which is later used for tuning convergence performance. We call the controller that makes (11) zero combined with the switching logic (12) symmetric walking control (SWC).

The idea of output zeroing of the function (11) is not new. Intuitively, in a steady walk, a human swings her leg opposite to the supporting leg. Some walking toys employ a similar concept, where only the hip "joint angles" can be constrained. The idea was also introduced in [20][21], where stability analysis via Poincaré map with an impulsive effect is performed. 
However, its systematic derivation from the viewpoint of global stability has not been done before. These are developed in the next section. Moreover, the obtained walking controller differs markedly from the past proposed ones in the following points. 1) We ensure global stability, not local stability. 2) We do not require Poincaré map nor its attendant stability analysis. 3) An invariance controller keeps the robot from falling 4) A passivity-based controller enables control energy and walking speed robustly, as explained in Section IV

\section{SWC Properties}

This section formulates SWC properties.

Proposition 3.1: Under (11) the controlled system (5) is reduced to the new 2-DOF Hamiltonian system with controlled Hamiltonian

$$
H_{c}(q, p)=\frac{1}{2 M_{c}} p^{2}+\left(m_{1}+2 m_{2}\right) g L \cos q,
$$

where $M_{c}=\left(m_{1}+m_{2}\right) L^{2}-2 m_{2} b L$.

Lemma 3.1: Let $F_{c}$ be the Hamiltonian flow associated with (13). $R$ is a reversing symmetry of $F_{c}$ under (11). Moreover, because of (12), the hybrid solution of the system (5) is bounded to the set $\mathcal{M}_{i}=\left\{x \in T^{*} \mathcal{M}|| q \mid \leq \bar{q}\right\}$. This lemma leads to one main result of this paper:

Theorem 3.1: Under (11) and (12) the following holds.

$$
\begin{aligned}
F i x\left(F_{c} \circ R\right) & =\mathcal{M}_{i 1}, \\
F i x\left(F_{c} \circ R \circ F_{c} \circ R\right) & =\mathcal{M}_{i 2},
\end{aligned}
$$

Therein, $\mathcal{M}_{i 1}$ and $\mathcal{M}_{i 2}$ are the semi-half subspaces of $\mathcal{M}_{i}$ defined as: $\mathcal{M}_{i 1}=\mathcal{M}_{i} \cap\{x|| p \mid>$ $\left.\sqrt{2 M\left(m_{1}+2 m_{2}\right) g L(1+\cos q)}\right\}$ and $\mathcal{M}_{i 2}=\mathcal{M}_{i} \cap\{x \mid$ $\left.|p|<\sqrt{2 M\left(m_{1}+2 m_{2}\right) g L(1+\cos q)}\right\}$.

Theorem 3.1 shows the global invariance of the solution in $\mathcal{M}_{i 1} \cup \mathcal{M}_{i 2}$. That is, any solution starting from $\mathcal{M}_{i 1} \cup \mathcal{M}_{i 2}$ lies on a symmetric orbit passing through the initial point! This invariance is the theoretical basis of passivity-based orbital stabilization developed in Section IV.

Therefore, the ideal walking gaits are generated. However, one important assumption is the realizability of $R$. Consider the dissipativity by the impulse equation at the leg exchange. The equation can be represented simply by a map ([6])

$$
R_{1}:\left(q_{1}, q_{2}, p_{1}, p_{2}\right) \mapsto\left(-q_{1},-q_{2}, \eta(q) p\right),
$$

in which $\operatorname{det}(\eta(q)) \leq 1$ (equality hold only for $q=0$ ). Hence, $R_{1}$ is not an involutive, but rather a contracting map. Therefore, the controlled Hamiltonian $H_{c}$ in (13) is found to be piece-wise constant and meets the passivity relationship.

$$
\frac{d H_{c}}{d t} \leq 0 \text {. }
$$

Thereby, we have obtained the second main result of the paper:

Theorem 3.2: If there is an energy-dissipation at leg exchange, the invariant set of SWC is hybrid $\omega$-limit set $L^{+}=$ $(\bar{q},-\bar{q}, 0,0) \cup(-\bar{q}, \bar{q}, 0,0)$, which is globally asymptotically stable on $\mathcal{M}_{i}$.

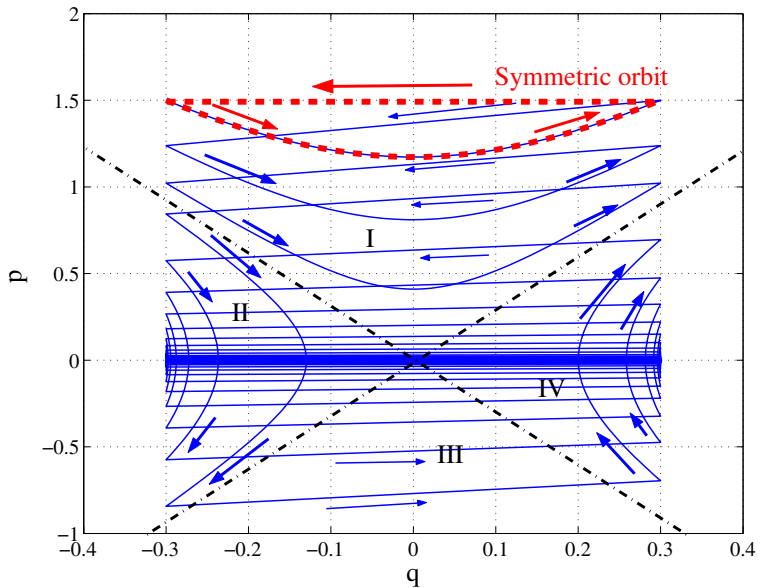

Fig. 4. Phase portrait of the reduced 2-DOF system under SWC with $\bar{q}=$ 0.3 . The solution starting from $(q(0), p(0))=(-0.3,1.5)$ asymptotically converges to the limit set (solid curve). If there is no dissipation at the leg exchange, it becomes a symmetric orbit (bold-dashed curve). The dash double-dot line indicates the separatrix.

Proof. From Lemma 3.1 the solution of the closed loop system is bounded. Take $H_{c}$ (13) as a positive semi-definite function. Then, Lemma 3.1 and LaSalle's invariance theorem [22] concludes the statement.

This is theoretical evidence of the statement in Section I-B; under SWC, the robot does not fall in principle. However, if we consider the slippage in contact between the foot and the ground, $\mathcal{M}_{i}$ should be narrowed appropriately, otherwise, one must consider the impulse reduction.

\section{Numerical illustration}

The phase portrait Fig. 4 illustrates Theorem 3.1 and Theorem 3.2. This figure shows the reduced (controlled) 2-DOF Hamiltonian flow $\phi_{t}$ of (13) with and without considering the impulsive effect. Note that the origin is an unstable equilibrium point (saddle). The separatrix and the switching border $|q|=\bar{q}$ divides the phase space $\mathcal{M}$ into $\mathcal{M}_{i 1}$ (region $\mathrm{I} \cup \mathrm{III}), \mathcal{M}_{i 2}($ region II $\cup \mathrm{IV})$.

In region I, $q$ is increasing monotonically (normal forward walking). If there is no impulse effect, the solution returns to the initial condition when it reaches the border. The orbit is the ideal walking gait corresponding to $R$-symmetric orbits. Note that the symmetric orbit is not isolated, but is instead surrounded by other symmetric orbits corresponding to different initial conditions (Recall the flow around a center equilibrium of a single autonomous system). This is a direct consequence of Theorem 3.1.

On the other hand, if the impulsive effect (16) at touchdown is considered, the momentum is decreased stepwise. The solution starting from region I eventually enters region II; the direction of motion is changed. Once this happens, the flow $\phi_{t}$ is "trapped" in region II $\cup$ IV. Then it finally converges to a $\omega$-limit set: $L^{+}=(\bar{q}, 0) \cup(-\bar{q}, 0)$. As the states become close to $L^{+}$, infinitely many switchings occur. This phenomenon is called Zeno [1]. Physically, the robot loses its kinetic energy 

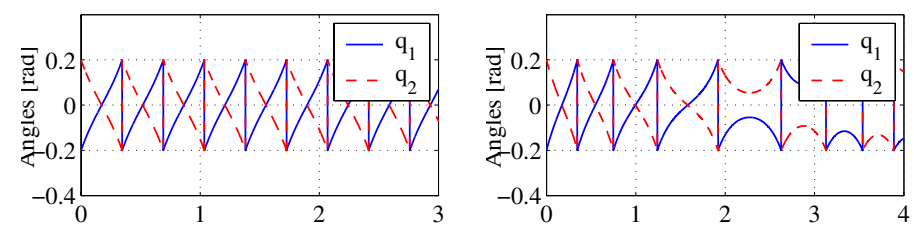

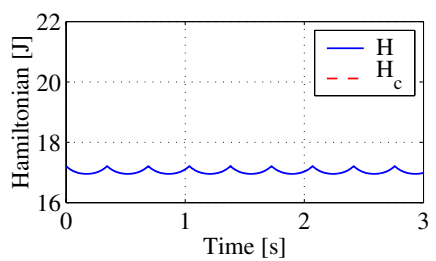

(a) Conservative

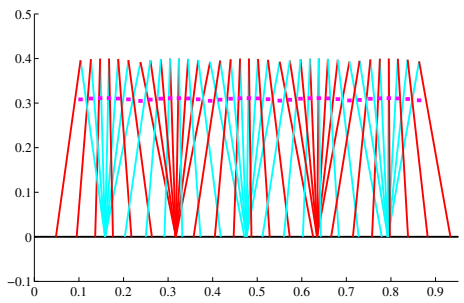

(c) Conservative

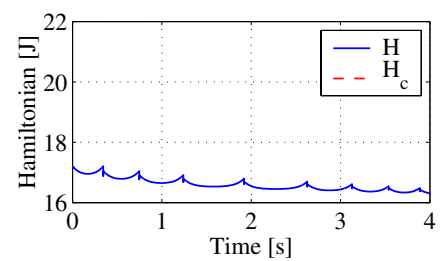

(b) Dissipative

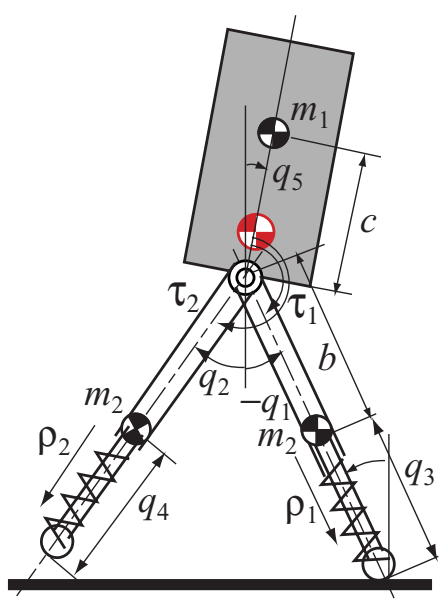

Fig. 6. Model of a five-link biped robot with torso: $\left(\rho_{1}, \rho_{2}\right)$ are the leg forces and $\left(\tau_{1}, \tau_{2}\right)$ are the hip torques.

into the system.

The additional DOF is installed as in Fig. 6, which shows the model of our planar biped walking/running robot Skipper II [11]. The new coordinates from the compass-model is the supporting leg length $\left(q_{3}, q_{4}\right)$ and the attitude of the torso $q_{5}$. Let $u=\left(\rho_{1}, \rho_{2}, \tau_{1}, \tau_{2}\right)$ be the control input.

In "walking mode", the leg length is kept constant during the supporting phase. Also, the torso inclination is kept constant. That is, the following output function is constrained to zero.

$$
y=h(x)=\left(q_{1}+q_{2}, q_{3}-r_{0}, q_{4}-r_{d}, q_{5}-q_{5 d}\right)^{T}
$$

Where $r_{0}$ is the natural length of the leg spring attached below the knee joint, which is further utilized for dynamic running motion [11]. The desired swinging leg length $r_{d}$ is designed simply so that the leg retracts or extends in proportion to $q_{2}$. Any retraction scheme that meets $r_{d}=r_{0}$ when $q_{2}=\bar{q}$ is possible for a robot that has light-weight feet, as Skipper II does.

\section{A. Speed control by leg extension}

First consider the simple impulsive control that compensates energy dissipation at touchdown. For simplicity, suppose that $c$ (torso length) $=0$. Then, one can realize the inverse map of $R_{1}$

$$
R_{2}:\left(q_{1}, q_{2}, p_{1}, p_{2}\right) \mapsto\left(q_{1}, q_{2}, \eta^{-1}(q) p\right),
$$

by exact (model-based) impulsive controller $\hat{u}$, which meets $R=R_{2} \circ R_{1}$.

Another option is a simple and robust control using the passivity of SWC. Thanks to the densely distributed symmetric orbit on $\mathcal{M}_{i}$, one can use the impulsive controller

$$
\hat{u}=k\left(\Gamma\left(\dot{x}-\dot{x}_{d}\right)\right) \quad \text { if } \dot{x}<\dot{x}_{d},
$$

where $k(\cdot)$ is an input transformation, $\Gamma>0$ is the gain, $\dot{x}$ represents the walking speed, and $\dot{x}_{d}$ is its commanded value. This controller does not require exact information on $\eta(q)$. 


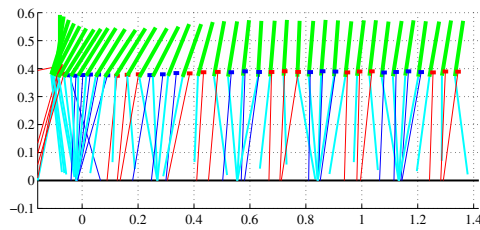

(a) Constant-speed regulation

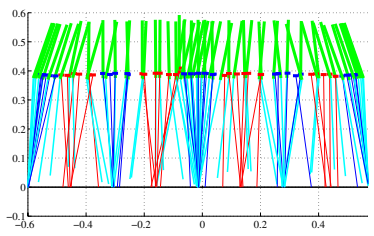

(b) Variable-speed tracking
Fig. 7. Animations of two kinds of speed-controlled walking. Only the right leg motion is depicted for visibility. (a): Constant speed walking with $\dot{x}_{d}=1.0 \mathrm{~m} / \mathrm{s}$. (b): Variable speed tracking with $\dot{x}_{d}=0.5 \rightarrow-0.5 \rightarrow 0.5$ $\mathrm{m} / \mathrm{s}$. the robot first moves forward, then turns backward and turns forward again. Thin solid lines indicate the virtual pendulum between ZMP and CoG (center of gravity). ZMP is shifted from the supporting point.
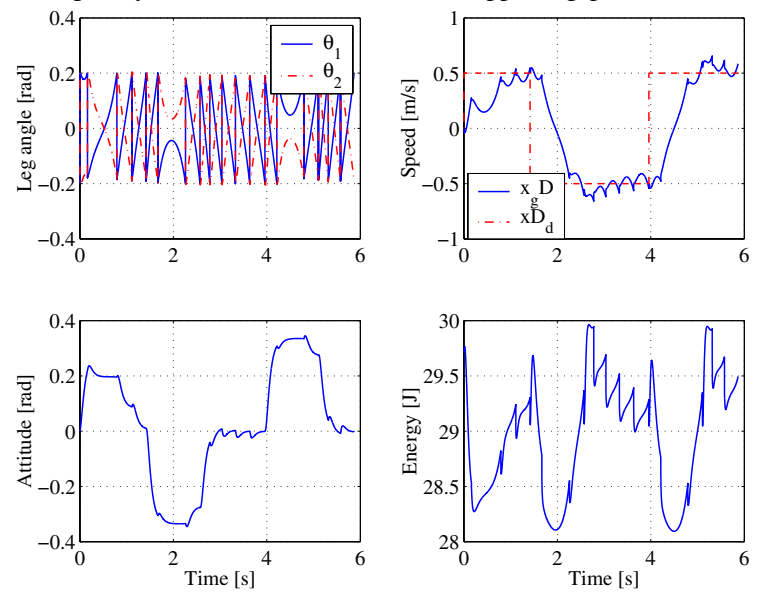

Fig. 8. Time evolution of the state variables corresponding to Fig. 7(b)

Also, steady state error can be removed by adding integral action.

\section{B. Speed control by torso inclination}

Non-zero inclination of the torso can be utilized for controlling the walking speed. Specifically, this paper proposes a controller of the proportional feedback form

$$
q_{5 d}=-\Gamma_{1}\left(\dot{x}-\dot{x}_{d}\right),
$$

where $\Gamma_{1}>0$ is the gain. With this controller, the input energy balances "automatically" with dissipated energy, which leads to a limit cycle. The steady state error can be removed by adding integral action.

The speed command $\dot{x}_{d}$ can be set arbitrarily. For example, Fig. 7 and Fig. 8 show simulation results of speedregulating/tracking walking. The robot starts from a standstill; then the torso pitches forward and the speed increases. As the speed approaches the desired value, the torso attitude converges to a constant value. Thereby, asymptotically stable walking gaits (limit cycles) with a specified walking speed are achieved.

There is a limitation in acceleration because of the boundedness of the torso angle. The effective maximum torso inclination is $\pm \pi / 2$, which results in the limitation of energy restoration. This affects the settling time of the walking speed. The longer the torso $\mathrm{CoG}$ is, the more energy can be restored during one walking cycle. It is also the same for larger $\bar{q}$, but large $\bar{q}$ leads to a high energy loss at touchdown, thereby increasing the steady state error of the walking speed.

\section{Application to 2D AND 3D BipEd PROTOTYPES}

\section{A. Simulation 1: Robustness against disturbance}

Before applying the controller to an actual robotic system, a precise 3D simulator was constructed on $L M S D A D S R$

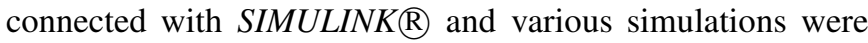
carried out. To make the controller robust, the simulation and later experiment use a simple PD-feedback.

$$
u=-K_{p} y-K_{d} \dot{y}
$$

Therein, $K_{p}>0$ and $K_{d}>0$ are relatively high gains.

As one example, Fig. 9 and Fig. 10 show a robustness test. In this simulation, the robot impacts with the wall during steady walking of $0.5 \mathrm{~m} / \mathrm{s}$. Despite this disturbance, the robot never falls. Instead, it merely stops. If the wall is removed, then it starts to walk again. Robustness comes from the invariance and the global stability of SWC (Theorem 3.1 and Theorem 3.2).

\section{B. Simulation 2: Symmetric 3D walking}

The SWC can be applied to a 3D walking model without any major modification. The only modification is the extension of the output function.

$$
y=h(x)=\left[\begin{array}{c}
q_{1}+q_{2} \\
q_{3}+q_{4} \\
q_{5}-r_{0} \\
q_{6}-r_{d} \\
q_{7}-q_{7 d} \\
q_{8}-q_{8 d}
\end{array}\right]
$$

Therein $\left(q_{1}, q_{2}, q_{3}, q_{4}\right)$ are the pitch and roll attitudes of the legs, $\left(q_{5}, q_{5}\right)$ are the leg lengths, and $\left(q_{7}, q_{8}\right)$ are the pitch and roll attitudes of the torso. The desired swinging leg length $r_{d}$ should be designed according to the attitude $\cos ^{-1}\left(\cos q_{2} \cos q_{4}\right)$. Figure 11 and 12 show one simulation result. Except for the orientation of the walking, which is difficult to maintain for the robot having point-contact foot, the stability and the behavior is the same as in the 2D walking model. However, because of the collision avoidance between the legs, some gain tuning is required.

\section{Experiment: Speed-controlled walking}

Because Skipper III is under preparation, only the experiments with Skipper II are shown here.

Figure 13 shows a typical gait of the symmetric walking. The robot stands on a tread mill. It is constrained to the sagittal plane by two transparent acrylic plates. It carries an attitude sensor comprising a gyro and an inclinometer at the torso; simple On/Off sensors at the feet detect the ground contact. In the current experimental setup, the walking speed is estimated from joint angles because position sensors are 


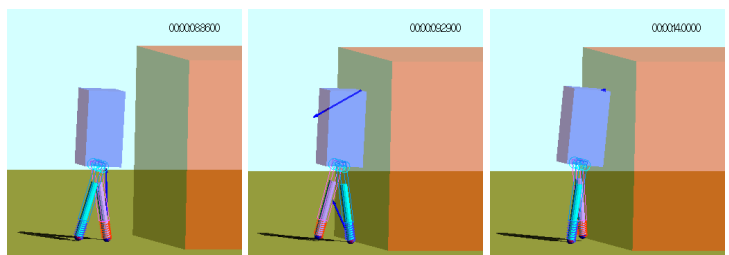

Fig. 9. A robustness test: during steady walking of $0.5 \mathrm{~m} / \mathrm{s}$, the robot impacts with the wall. However, the robot does not fall. It merely decreases the energy and stops. If the wall is removed, then it begins to walk again.
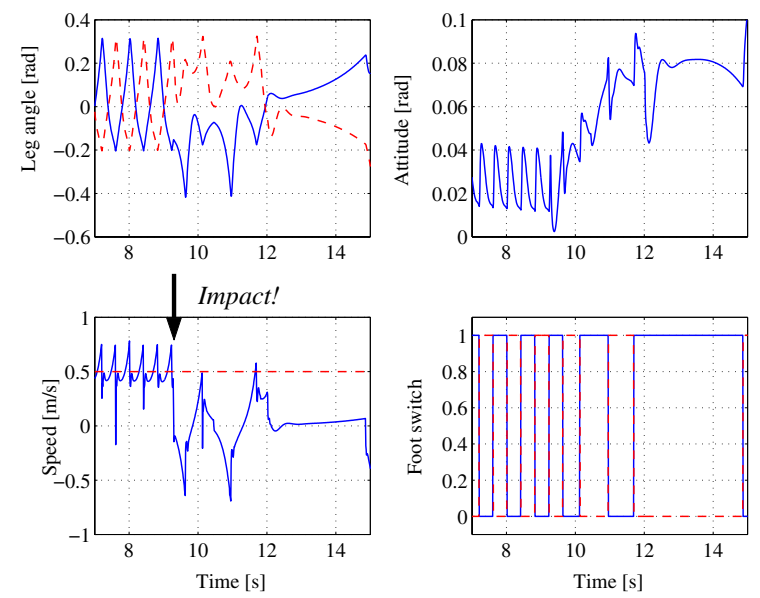

Fig. 10. Time evolutions corresponding to Fig. 9: After impact at $9.23 \mathrm{~s}$, the walking speed decreases and finally converges to zero around $12.5 \mathrm{~s}$ (left bottom). Because the commanded speed is fixed to $0.5 \mathrm{~m} / \mathrm{s}$ in this example, the torso attitude still increases (right top).

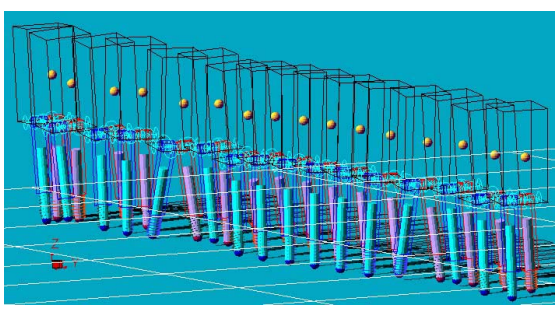

Fig. 11. 3D animation of symmetric walking.
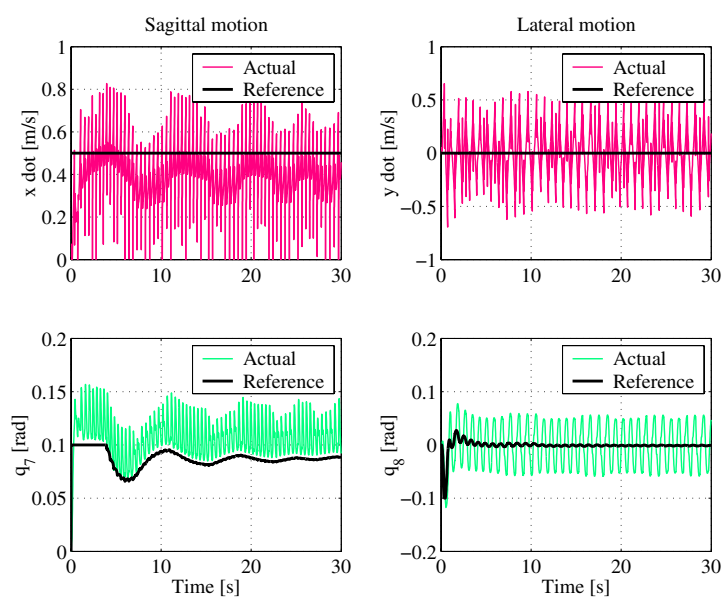

Fig. 12. Time evolution of the state variables during 3D symmetric walking. The commanded walking speed is $0.5 \mathrm{~m} / \mathrm{s}$ to the forward direction.
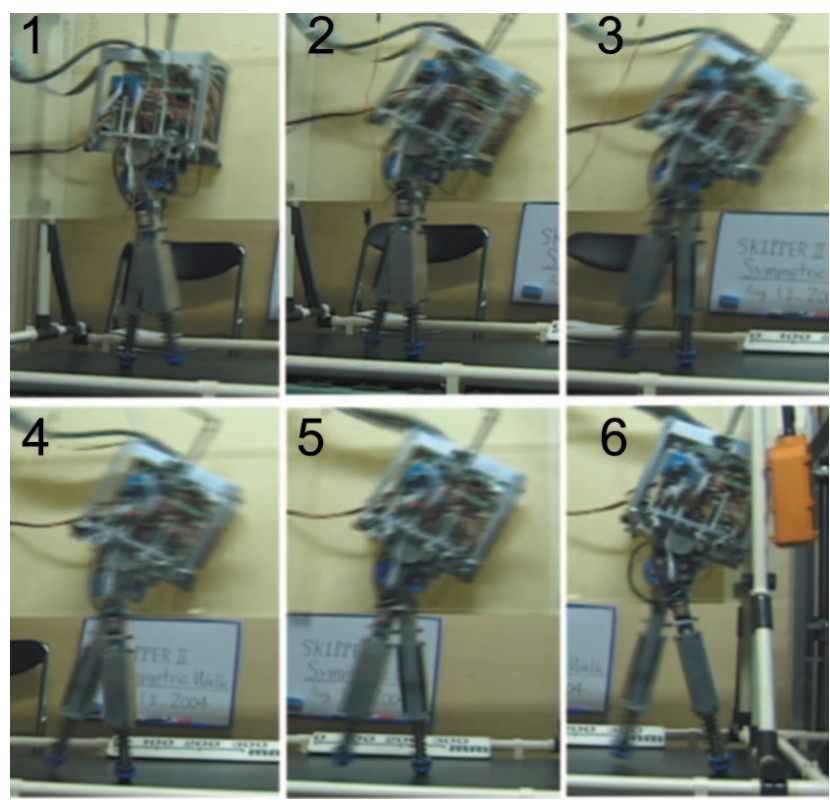

Fig. 13. Snap-shot of the walking experiment: The robot initially stands still. When the speed command is set to some positive value, the torso pitches forward and then starts to walk.
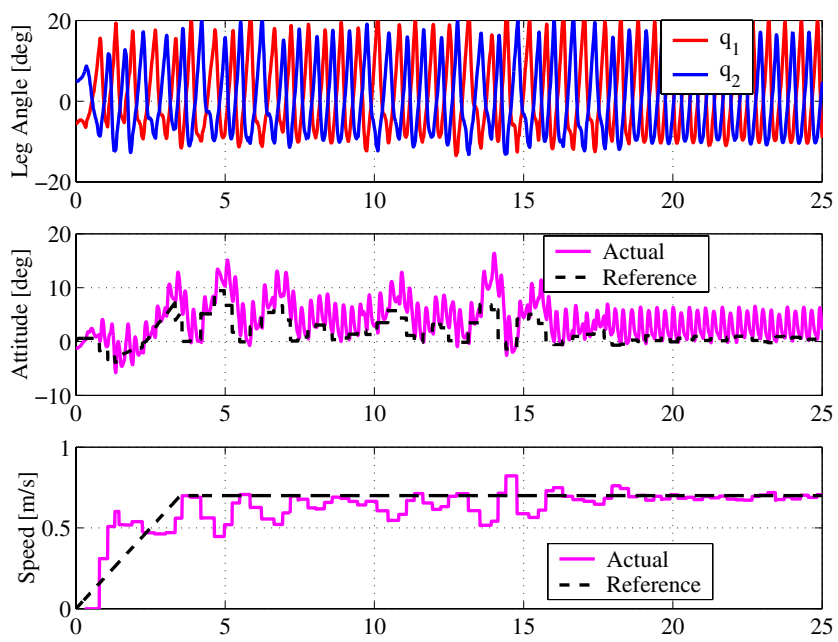

Fig. 14. Time evolution of some state variables during a long-distance and high-speed walking experiment on tread mill. The commanded speed is a ramp signal of $0-0.75 \mathrm{~m} / \mathrm{s}$. Asymmetry in the top graph comes from the slippage of the legs and the dely of the PD-feedback.

not installed. Figure 14 shows the time evolution of the main state variables during long-distance and high-speed walking experiments. The bottom graph indicates that the error is very small. One can start a walking experiment from any initial conditions. In this experiment, a variable feedback gain $\Gamma_{1}=\left|\dot{x}-\dot{x}_{d}\right|$ and variable step length $\bar{q}=\Gamma_{2}\left|\dot{x}-\dot{x}_{d}\right|$ are introduced to improve tracking performance. Because the behavior is almost identical to that in simulations, we do not repeat their explanations. But note that the information regarding physical parameters such as mass distribution or length is not required to obtain a stable limit cycle because 
SWC is not a conventional model-based controller.

\section{DISCUSSION}

\section{A. Advantages of SWC}

The SWC combined with passivity-based controller allows restoration of the total mechanical energy and control of the walking speed. Solutions starting from any point in the domain $\mathcal{M}_{i}$ converge globally to a stable limit cycle or stable hybrid $\omega$-limit sets. Global stability was proved theoretically using the invariance principle. The controller is written in a program of very small size: it can be easily implemented on an inexpensive micro-controller. Moreover, the control parameter is actually the switching angle $\bar{q}$ only. There is no room for "trial-and-error". The SWC has strong passivity and robustness against disturbance. That passivity is especially useful for human-safety functions, which conventional trajectory-based controllers do not support. For example, when the desired speed is set to zero, the robot can stop even if an unknown disturbance is applied. The settling time depends on the magnitude of the disturbance. Alternatively, the disturbance can be transferred to the propulsion energy of walking: a sort of an externally-driven walking just like pushing a tire on the floor. The SWC can be utilized as an "auto balancer" of bipedal humanoid robots, which may be activated at the lowest layer of the whole control system.

Its strong stability and robustness will encourage application of SWC to many kinds of bipedal robot without major modifications. We have succeeded in simulating the stable 3D walking of Skipper III. We have also succeeded in simulating stable walking of a five-linked planar anthropomorphic biped robot with a "passive knee joint" using an inexpensive fourline controller in which the mechanical energy is compensated not only by the torso inclination, but also by the ankle torque at the double support phase [8].

\section{B. Road to running: Impulse reduction, input optimization}

Energy loss attributable to the impact is unavoidable in stiff walking. This is a direct consequence of the impact equation (16). However, the energy loss can be reduced if the leg is compliant. Moreover, kinetic energy can be restored into the spring mechanism. Compliant leg actuation with a spring mechanism leads to energy preserving symmetric running controller implemented in [11]. We have obtained speed controlled walking by torso inclination. For that reason, we expect that speed-controlled running can be achieved in the same context.

In this regard, the transition from walking to running will be an interesting problem. Our precise simulations showed that, for walking faster than $1.33 \mathrm{~m} / \mathrm{s}$, which corresponds to Froude number $=\dot{x}_{G}^{2} /\left(g L_{G}\right)=0.45$, the robot slipped on the ground; thereby, a very short period of flight occurred. This is almost the same conclusion of a preferable walk-to-run transient speed, similar to that discussed in biomechanics studies (e.g. [23]). Faster locomotion without slipping is possible only if a sufficient ground reaction force is provided by an "extending leg". In this case, the mechanical energy is restored not only by leg extension, but also by torso inclination. Even when some ankle torque is available, because of the ZMP limitation, we ultimately rely on leg extension to increase the energy. This phenomenon leads to hip-torque reduction. We expect optimal walking and running gait to be realized using a "single controller". All these expectations will be tested during experiments using Skipper prototypes.

\section{REFERENCES}

[1] A. Matveev and A. Savkin, Qualitative theory of hybrid dynamical systems, Control Engineering, Birkhauser, 2000.

[2] J. Yamaguchi, A. Takanishi and I. Kato, "Development of a biped walking robot compensating for three-axis moment by trunk motion," Proc. IEEE/RSJ IROS, 1993, pp. 561-566.

[3] S. Hyon, D. Aoyagi, S.A. Setiawan, J. Yamaguchi and A. Takanishi, "Physical interaction between a human and a bipedal humanoid robot realization of human-follow walking -," Proc. RSJ Annual Conf., 1998, pp. 951-952 (in Japanese).

[4] K. Hirai, M. Hirose, Y. Haikawa and T. Takenaka, "The development of the Honda Humanoid Robot," Proc. IEEE ICRA, 1998, pp. 1321-1328.

[5] S. Kajita, F. Kanehiro, K. Kaneko, K. Fujiwara, K. Harada, K. Yokoi and H. Hirukawa, "Resolved momentum control: humanoid motion planning based on the linear and angular momentum," Proc. IEEE/RSJ IROS, 2003, pp.1644-1650.

[6] T. McGeer, "Passive dynamic walking," International Journal of Robotics Research, vol. 9, no. 2, 1990, pp. 62-82.

[7] M.W. Spong, "Passivity-based control of the compass gait biped," Proc. IFAC World Congress, 1999, pp. 19-23.

[8] S. Hyon and T. Emura, "Symmetric walking control and its application to five-linked anthropomorphic biped," Proc. SOBIM Annual Conf., 2004, pp. 207-210, (in Japanese).

[9] S. Hyon, Development and Control of One-Legged Running Robot, Ph.D Thesis, Tokyo Institute of Technology, 2002.

[10] S. Hyon and T. Emura, "Energy-preserving control of passive onelegged running robot," Advanced Robotics, vol. 18, no. 4, 2004, pp. 357-381.

[11] S. Hyon and T. Emura, "Running control of a planar biped robot based on energy-preserving strategy," Proc. IEEE ICRA, 2004, pp. 3791-3796.

[12] J. Lamb and J. Roberts, "Time-reversal symmetry in dynamical systems: A survey," Physica D, vol. 112, 1998, pp. 1-39.

[13] W. Schwind and D.E. Koditschek, "Control of forward velocity for a simplified planar hopping robot," Proc. of IEEE ICRA, 1995, pp.691696.

[14] R. Altendorfer, D.E. Koditschek and P. Holmes, "Toward factored analysis of legged locomotion models," Proc. IEEE ICRA, 2003, pp. 37-44.

[15] H. Nijmeijer and A.J. vd. Schaft, Nonlinear Dynamical Control Systems, Springer, 1990.

[16] V.I. Arnold, Mathematical methods of classical mechanics, Springer, 1978.

[17] S. Hyon, T. Emura and T. Ueta, "Detection and stabilization of hybrid periodic orbits of passive running robots," Proc. Mechatronics and Robotics, 2004, pp.1309-1314.

[18] S. Morita, H. Fujii, T. Kobiki, S. Minami and T. Ohtsuka, "Gait generation method for a compass type walking machine using dynamical symmetry," Proc. IEEE/RSJ IROS, 2004, pp. 2825-2830.

[19] J. Hale, Ordinary Differential Equations, Wiley, 1969.

[20] R.Q. vd. Linde, "Passive bipedal walking with phasic muscle contraction", Biol. Cybern., vol. 81, no. 3, 1999, pp.227-237.

[21] J. Grizzle, G. Abba and F. Plestan, "Asymptotically stable walking for biped robots: Analysis via systems with impulse effects," IEEE Trans. Automatic Control, vol. 46, 2001, pp. 51-64.

[22] H. Khalil, Nonlinear Systems, 2nd ed. Prentice Hall, 1996.

[23] R. Kram, A. Domingo and D. Ferris, "Effect of reduced gravity on the preferred walk-run transition speed," J. Experimental Biology, vol. 200, 1997, pp. 821-826. 